\title{
Existence of a Tribo-Modified Surface Layer on SBR Elastomers: Balance Between Formation and Wear of the Modified Layer
}

\author{
Milad Mokhtari • Dik J. Schipper • Nadia Vleugels • \\ Jacques W. M. Noordermeer
}

Received: 29 December 2014/ Accepted: 15 February 2015/Published online: 22 March 2015

(c) The Author(s) 2015. This article is published with open access at Springerlink.com

\begin{abstract}
In most of the tribological contacts, the composition and tribological properties of the original interface will change during use. The tribo-films, with modified properties compared to the bulk, are dynamic structures that play a significant role in friction. The existence of a tribo-modified surface layer and its importance on the overall friction of elastomers has been shown both theoretically and experimentally before. The characteristics of the modified surface layer deserve specific attention since the tribological properties of elastomers in contact with a rough counter-surface are determined by these modified surfaces together with the properties of bulk of the material. Both the formation of the modified layer and the break down (wear) of it are of importance in determining the existence and thickness of the tribo-modified layer. In this study, the importance of the wear is emphasized by comparing two styrene butadiene rubber-based elastomers in contact with a granite sphere. A current status of perception of the removal and the stability of the modified surface layers on rubbers is introduced as well as experimental work related to this matter and discussion within literature. Pin-on-disk friction tests are performed on two SBR-based samples in contact with a granite sphere under controlled environmental conditions to form the modified surface layer. Although the hysteresis part of the
\end{abstract}

M. Mokhtari ( $\square)$. D. J. Schipper

Surface Technology and Tribology, Faculty of Engineering

Technology, University of Twente, P.O. Box 217,

7500 AE Enschede, The Netherlands

e-mail: m.mokhtari@utwente.nl; mokhtari.ac@gmail.com

N. Vleugels · J. W. M. Noordermeer

Elastomer Technology and Engineering, Faculty of Engineering

Technology, University of Twente, P.O. Box 217,

7500 AE Enschede, The Netherlands friction force which has a minor contribution in the overall friction is not markedly different, the total measured friction coefficient differs significantly. Mechanical changes both inside and outside the wear track are determined by atomic force microscope nano-indentations at different timescales to examine the modified surface layer on the test samples. The specific wear rates of the two tribo-systems are compared, and the existence of the modified surface layer, the different measured friction coefficient and the running-in distances toward steady-state friction are explained considering different wear rates. A conceptual model is presented, correlating the energy input into the tribo-system and the existence of a modified surface layer.

Keywords Friction-modified surface layer - Balance between formation and wear . SBR - AFM nanoindentation $\cdot$ Rubber friction
List of symbols
$F_{\mathrm{f}} \quad$ Total friction force $(\mathrm{N})$
$F_{\text {vis }} \quad$ Hysteresis contribution of friction force induced by viscoelastic losses $(\mathrm{N})$
$\tau_{\mathrm{f}} \quad$ Frictional shear stress $(\mathrm{Pa})$
$A_{\text {real }} \quad$ Real area of contact $\left(\mathrm{m}^{2}\right)$
$\mu_{\mathrm{f}} \quad$ Total coefficient of friction
$F_{\mathrm{N}} \quad$ Nominal normal load $(\mathrm{N})$
$\sigma_{0} \quad$ Nominal contact pressure $(\mathrm{Pa})$
$A_{0} \quad$ Nominal area of contact $\left(\mathrm{m}^{2}\right)$

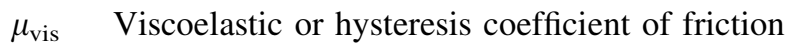
$P(q) \quad$ Real to nominal area of contact ratio as a function of wave vector
$\omega \quad$ Frequency of the applied load to the rubber $(\mathrm{rad} / \mathrm{s})$
$\lambda \quad$ Length scale of the roughness under study $(\mathrm{m})$
$q \quad$ Amplitude of the roughness wave vector $(1 / \mathrm{m})$ 
$q_{0} \quad$ Lower wave-vector cutoff corresponding to the longest wave length of roughness $(1 / \mathrm{m})$

$q_{1} \quad$ Higher wave-vector cutoff corresponding to the shortest wave length of roughness $(1 / \mathrm{m})$

$C(q)$ Power spectral density of the roughness $\left(\mathrm{m}^{4}\right)$

$A(q)$ Apparent contact area when the surface is smooth on all wave vectors $>q\left(\mathrm{~m}^{2}\right)$

$\phi \quad$ Angle between the velocity vector and the wave vector $\overrightarrow{\boldsymbol{q}}$ (rad)

E Modulus of elasticity $(\mathrm{Pa})$

$v \quad$ Poisson's ratio

$a_{\mathrm{T}} \quad$ The temperature-frequency viscoelastic horizontal shift factor

$\zeta \quad$ Magnification factor

$E_{\text {in }} \quad$ The tribological energy input (J)

$s \quad$ The sliding distance $(\mathrm{m})$

$Q_{\mathrm{f}} \quad$ The formation rate of the modified surface layer $(\mathrm{kg} / \mathrm{s})$

$Q_{\mathrm{w}} \quad$ The wear rate $(\mathrm{kg} / \mathrm{s})$

$k \quad$ The specific wear rate $\left(\mathrm{mm}^{3} / \mathrm{Nm}\right)$

$\delta_{\text {total }}$ The thickness of the modified surface layer at balance (m)

$\delta_{\mathrm{f}} \quad$ The thickness of the modified surface layer just due to formation, neglecting wear (m)

$\delta_{\mathrm{w}} \quad$ The thickness of the worn layer (m)

\section{Introduction}

Friction needs to be accurately accounted for in a smart design of various rubber engineering components including but not limited to tires, rubber seals, wiper blades, conveyor belts and syringes [1-4]. Even today, there remains an incomplete understanding of the rubber friction problem, in spite of the fact that great interest has been dedicated by studying the tribological behavior of rubber sliding contacts over the last 50 years, which marks the difficulty of the problem.

Classifying the friction force between rubber and a rough surface, two main contributors are commonly described, i.e., the adhesion component and the hysteresis component [5]. Adhesion is related to the attractive forces between the contacting bodies [6]. Cyclic deformation of the rubber dissipates energy via the internal damping in the bulk of the material and generates the hysteresis component of friction [2]. Other contributors to rubber friction are energy dissipation due to crack opening [7] and energy dissipation in shearing of a thin viscous film [8]. The significant role of interfacial interactions in determining the wet sliding friction of elastomer compounds has been noted by Pan [9]. The friction force contributions mentioned before are summarized in terms of two main forces: (1) the contributions related to the viscoelastic deformation of the rubber, and (2) the contributions related to the real area of contact as defined in Eq. (1). One cannot indicate one contributor as the main contributor to the friction as a generalized rule, but depending on the tribological conditions, hysteresis or contribution from the real area of contact can play a dominant role in determining the overall friction.

$F_{\mathrm{f}}=F_{\text {visc }}+\tau_{\mathrm{f}} A_{\text {real }}$

where $F_{\mathrm{f}}, F_{\text {visc }}$ are the forces concerning the total friction and the contribution from the hysteresis losses, respectively, and the product $\tau_{\mathrm{f}}, A_{\text {real }}$ represents the force in the real area of contact where $\tau_{\mathrm{f}}, A_{\text {real }}$ are the frictional shear stress and real area of contact.

\subsection{Contact and Friction of Rubbers}

Several contact models have been proposed and examined for various materials [2, 10-12]. Among them, the asperity contact theory, first addressed by Greenwood and Williamson [10], has attracted attention of several researchers for a long time and has been used to describe rough surfaces in contact. Neglecting the interaction between neighboring asperities is the main disadvantage of such models. Rubbers are considered as elastically soft materials that are flexible and, therefore, can deform much easier than most of the engineering materials such as metals. Thus, the effect of the asperities on each other cannot be neglected. The interaction between the asperities can be added to the current asperity models [13, 14]; however, these approaches remain quite approximate [15]. On the other hand, Persson's contact theory does not pre-exclude any scale of roughness (unlike asperity contact models) from the contact analysis [2], it considers the contact in the limit of full contact and further extends the analysis to the partial contact by imposing a boundary condition. Although Manners and Greenwood [16] raise some concerns about the boundary conditions applied in Persson's theory, the rubber behavior (a hyperelastic material with the ability to bend and fill out the roughness on at least small wave lengths) is more analogous to Persson's analysis, than the asperity contact models (where it is assumed that contact occurs on segregated islands, far from each other, which are named asperities and do not have any influence on each other because of the far distances in between).

However, Persson's contact theory as like as other models is an approximation (because of the assumptions which are made to simplify the complex problem of the contact between rubber-like materials and rigid rough surfaces) to the physical reality that occurs. It has been 
subject to comparison with other contact models and has been analyzed [17] extensively. The results of comparisons can lead to guidelines for enhancing the theory.

The basic equations of the hysteresis coefficient of friction as well as the real area of contact, based on Persson's contact and friction theory, are summarized below [7]:

$\mu_{\mathrm{vis}} \approx \frac{1}{2} \int_{q_{0}}^{q_{1}} \mathrm{~d} q q^{3} C(q) P(q) \int_{0}^{2 \Pi} \mathrm{d} \phi \cos \phi \operatorname{Im}\left[\frac{E(q v \cos \phi)}{\left(1-v^{2}\right) \sigma_{0}}\right]$,

where the function $P(q)=A(q) / A_{0}$ is given by

$P(q)=\frac{2}{\Pi} \int_{0}^{\infty} \mathrm{d} x \frac{\sin x}{x} \exp \left[-x^{2} G(q)\right]$

where

$G(q)=\frac{1}{8} \int_{q_{0}}^{q} \mathrm{~d} q q^{3} C(q) \int_{0}^{2 \Pi} \mathrm{d} \phi\left|\frac{E(q v \cos \phi)}{\left(1-v^{2}\right) \sigma_{0}}\right|^{2}$.

The contribution to the overall friction due to the force of shearing a thin fluid-like film formed by segments of rubber molecules [8] has been suggested to be modeled as:

$F_{\text {shear }}=\tau_{\mathrm{f}} A$ with $\tau_{\mathrm{f}}=\tau_{0}\left(a_{\mathrm{T}} v\right)^{\alpha}$

where $a_{\mathrm{T}}$ is the temperature-frequency viscoelastic shift factor and $\tau_{0}$ is basically a fitting parameter. One can find more about the origin of the formulas in [7].

The present study investigates the importance of shearing the modified surface layer and its contribution to the overall friction, and highlights the nature of a dynamic process which involves formation and removal of the modified surface layer.

\subsection{Thin Modified Surface Layer}

The composition and tribological properties of the original interface changes during use in most tribological contacts. This has been shown for various materials under various tribological conditions [18]. However, there are not many studies on the existence and the properties of the modified surface layer in contact with elastomers with a rigid rough counter-surface. It is worth emphasizing that these modified surfaces play an important role in determining the tribological behavior. The important role of shearing the top rubber layer in the overall friction is demonstrated in some tribological systems [19]. It has been shown that $\tau_{\mathrm{f}}$ may change due to degradation of the top rubber layer in contact with the counter-surface, and therefore, the overall friction changes [20]. The rubber surface that is in contact with the counter-surface undergoes changes in mechanical properties (and chemical compositions) in comparison with the bulk or with the non-contact parts of the rubber surface [21-27]. Therefore, the mechanical properties of the modified surface layer should be used in modeling the friction and not the properties of the bulk of the material (see Fig. 1). This is especially important in tribo-systems where the contribution from shearing the top rubber layer is controlling the friction [20]. Moreover, not much research has been performed on understanding the physical properties of the tribo-modified surface layers on elastomers. In addition, the dynamics of the formation, removal and the stability of the tribo-modified surface layers on rubbers (which is the result of a balance between formation and wear of the modified surface layer) are still not well understood. The formation process (and rate) of the modified layer is not discussed in the present study. However, the modified layer is also subject to wear. The generated modified surface layer might be completely gone due to wear if the wear rate is equal to or higher than the modification rate. The generated modified surface layer, i.e., the mechanical properties, is studied experimentally. The mechanical properties of the modified surface layers are dependent on the tribological conditions; it has been shown that a more severe tribological condition might lead to more loss of elastic modulus [20]. Moreover, wear is also dependent on the tribological conditions, and it can change dramatically depending on the tribological conditions. Therefore, the friction behavior, wear and tribo-film formation are interrelated for the studied tribo-systems.

Gaining a quantitative insight into the interfacial layer existence and the change in properties demands a detailed study of several tribo-systems. The present study focuses on the important role of wear on the existence of such a layer.

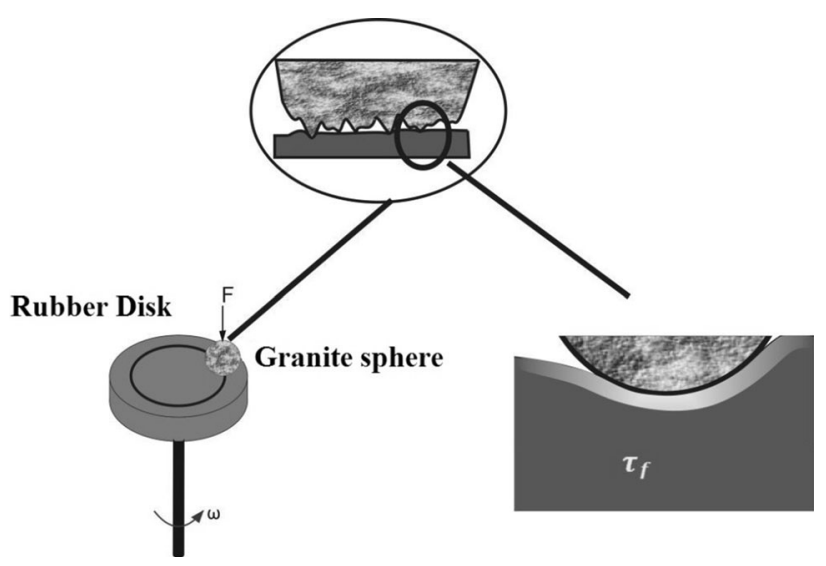

Fig. 1 Rough granite ball is shown in contact with a rubber disk. The contact is considered on different scales. Viscoelastic losses together with shearing of the granite to the rubber determine the friction. Shear occurs between a modified surface layer and rough granite 


\section{Materials and Experimental Methods}

\subsection{Materials}

The rubber formulations employed in this study are based on a solution-polymerized styrene butadiene rubber (SSBR Buna VSL VP PBR 4045 from Lanxess, Leverkusen, Germany), type of elastomer with a styrene content of $25 \%$, a vinyl content of $25 \%$ and a butadiene content of $50 \%$. Two samples are prepared. Sample (1) is unreinforced vulcanized SBR rubber, and sample (2) is reinforced with 3 parts per hundred rubber (phr) aramid fibers. The fibers which were supplied by Teijin Aramid BV, the Netherlands, have an initial length of $3 \mathrm{~mm}$ and a fiber diameter of 10-12 microns. Moreover, 3-octanoylthio-1propyltriethoxysilane (NXT) from Evonik $\mathrm{GmbH}$ is used as coupling agent for sample (2) to provide sufficient chemical bonding between the rubber matrix and the fibers. The ethoxy part of the used coupling agent molecule reacts with the fibers, while the thiol part goes through a reaction with the rubber. In order to enhance the fiber/rubber adhesion, the fibers were coated with an epoxy-amine coating. No coupling agent was used in preparation of the first sample. The fibers were randomly oriented, so the mechanical properties do not differ along different axes. Moreover, the fibers were selected to be sufficiently thin and closely spaced, so that the materials appear homogeneous on the length scales which matter for friction. An overview of the rubber compounds prepared with the corresponding amounts (phr) of the components is given in Table 1.

The compounds were prepared on a $350-\mathrm{mL}$ Brabender $350 \mathrm{~S}$ internal mixer using a two-stage mixing procedure $\left(50{ }^{\circ} \mathrm{C}, 1: 1.13\right.$ rotor speed ratio); the fibers and the zinc oxide are added to the loaded rubber after 1 and $2.5 \mathrm{~min}$, respectively. Then, after $3 \mathrm{~min}$, the residue is swept back in the hopper. The fibers are dispersed on a Polymix 80T mill

Table 1 Rubber formulation of prepared samples with the amounts of components indicated in weight parts of component per hundred weight parts of the rubber (phr)

\begin{tabular}{lll}
\hline Compound & Sample 1 & Sample 2 \\
\hline Description & phr & phr \\
S-SBR & 100 & 100 \\
Aramid fiber & 0 & 3 \\
NXT & 0 & 1 \\
Sulfur & 1.4 & 1.4 \\
ZnO & 2.5 & 2.5 \\
Stearic acid & 2.5 & 2.5 \\
TBBS & 1.7 & 1.7 \\
\hline
\end{tabular}

${ }^{a} \mathrm{~N}$-tert-butylbenzothiazole-2-sulphenamide is used as accelerator for $30 \mathrm{~min}$ at $130{ }^{\circ} \mathrm{C}$. After the dispersion of the fibers on the mill, the first-stage master batch is returned to the mixing chamber for a second stage and mixed with the curatives up to a temperature of $100{ }^{\circ} \mathrm{C}$ at $75 \mathrm{rpm}$ for $3 \mathrm{~min}$.

The compounds are then vulcanized in a Wickert press WLP 1600 under a pressure of 100 bar and at $160{ }^{\circ} \mathrm{C}$, according to their $\mathrm{t} 90+2$ min optimum vulcanization time, as determined in a Rubber Process Analyzer RPA 2000 of Alpha Technologies, following the procedure described in ISO 3417.

\subsection{Viscoelasticity of Compounds}

The dynamic properties of the rubber samples were measured by Dynamical Mechanical Analysis in a Metravib DMA2000 dynamic spectrometer in temperature sweep mode, under dynamic and static strains of 0.1 and $1 \%$, respectively, at a fixed frequency of $10 \mathrm{~Hz}$, over a wide temperature range $\left(-90\right.$ to $\left.+120{ }^{\circ} \mathrm{C}\right)$ with strip specimens of $2 \mathrm{~mm}$ thickness and $35 \mathrm{~mm}$ length to determine the glass transition temperatures of the samples. The measured loss tangent, which is the ratio between the loss and storage moduli, is shown as a function of temperature for the two samples in Fig. 2.

In order to create dynamic mechanical master curves, frequency-dependent elasticity modulus measurements in tension mode were taken at different temperatures between -30 and $50{ }^{\circ} \mathrm{C}$, each varying the frequency between 1 and $200 \mathrm{~Hz}$. The glass transition temperature and the selected reference temperature $T_{\text {ref }}=27^{\circ} \mathrm{C}$ are used to shift the measured elastic modulus $E(\omega)$ versus frequency $\omega$ both horizontally and vertically. The calculated master curves for the moduli of elasticity as a function of frequency are shown in Fig. 3 for both samples. The reinforced sample (2) has a higher elasticity modulus than sample (1) but the mechanical characteristics of the two samples are not grossly different, especially at higher frequencies. The loss tangent which has been used traditionally as a measure for the hysteresis in the rubber samples is approximately similar for lower temperatures; however, sample (1) shows higher values for higher temperatures, see Fig. 2.

\subsection{Friction Tests}

The friction between the prepared rubber disks and a granite ball with a diameter of $30 \mathrm{~mm}$ and a root-meansquare roughness of $2.1 \mu \mathrm{m}$ was measured by a ball-ondisk setup under controlled environmental conditions: The temperature is kept constant at $27{ }^{\circ} \mathrm{C}$ and the relative humidity at $50 \%$. The sliding velocity is $5 \mathrm{~mm} / \mathrm{s}$, and the nominal contact pressure between the granite and the rubbers is $0.175 \mathrm{MPa}$. The roughness of the granite sphere 
Fig. 2 Measured loss tangent as a function of temperature for both samples

Fig. 3 Shifted storage (top) and loss (bottom) moduli of elasticity as a function of frequency
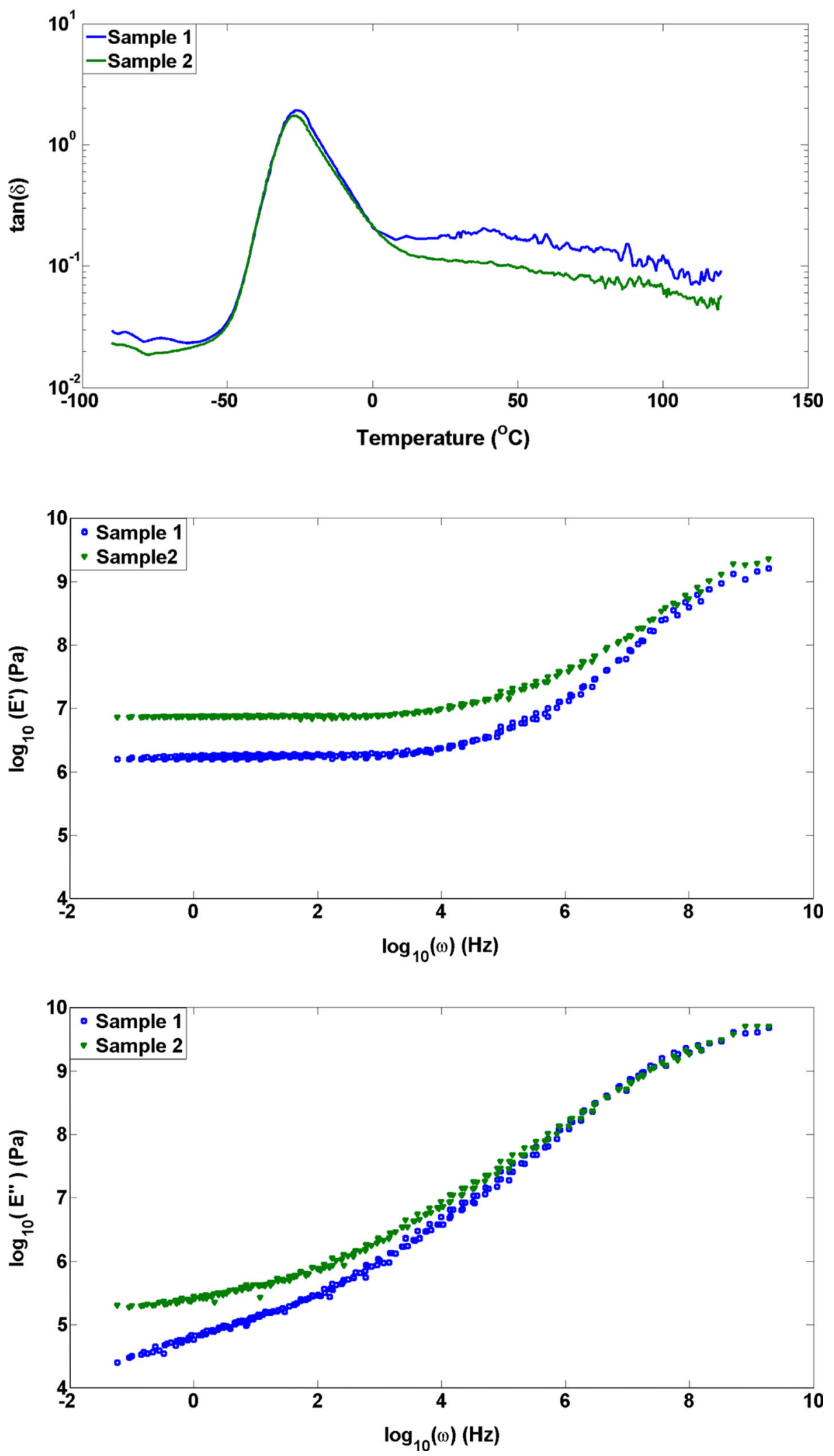

was measured using two different scanning techniques, confocal microscopy and atomic force microscopy (AFM) in the contact mode. The measurements obtained by both techniques as well as the calculated power spectral density of the roughness of the granite ball governed by each method are shown in Fig. 4. The surface of the granite ball 

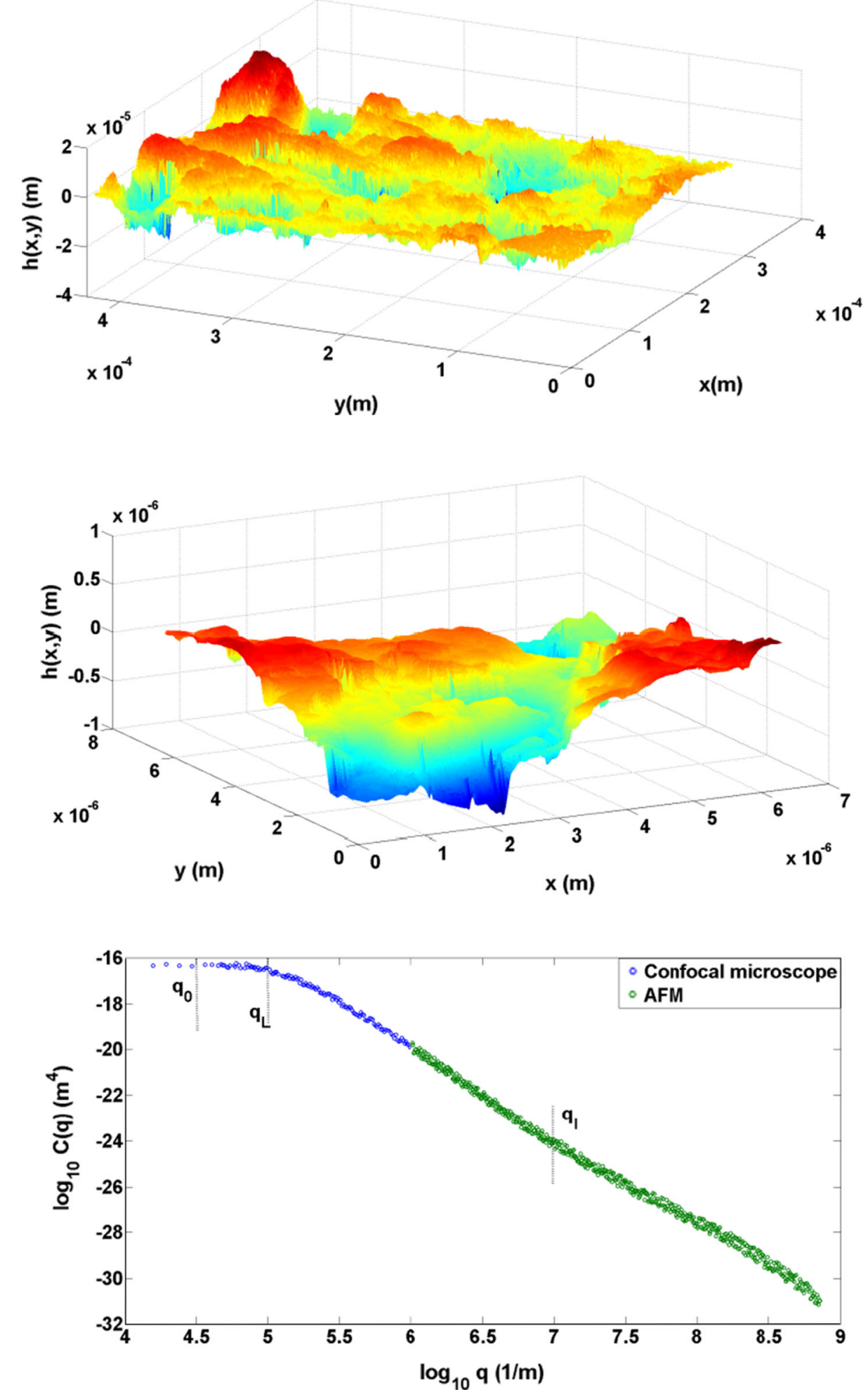

Fig. 4 Rough contact surface obtained by use of a confocal microscope in the wave-vector range $1.58 \times 10^{4} \mathrm{~m}^{-1} \leq q \leq 9.77 \times$ $10^{5} \mathrm{~m}^{-1}$ (top), by use of an atomic force microscope in the range
$9.77 \times 10^{5} \mathrm{~m}^{-1} \leq q \leq 7.07 \times 10^{8} \mathrm{~m}^{-1}$ (middle) and the calculated power spectral density of the roughness (bottom) 
is prepared by sand blasting. As shown, the granite surface is self-fractal. It is noteworthy that confocal microscopy and atomic force microscopy measurements provide consistent measures.

The slope of the power spectrum in the self-affine fractal region corresponds to the Hurst exponent $H=0.87$ or fractal dimension $D_{\mathrm{f}}=3-H=2.13$ that is very typical for most surfaces [28]. The measured steady-state coefficient of friction under dry conditions is shown in Fig. 5 (blue bars). In addition, the contribution from the hysteresis component of friction is decoupled from the total friction and measured by a simple experiment; the rubber surface was wetted by a very thin layer of oil (Ondina 927 with a dynamic viscosity of 78 $\mathrm{mPas}$ at $20^{\circ} \mathrm{C}$ ) such that the lubricated tribo-system remains in the boundary lubrication regime. The measured hysteresis contribution to the total friction is also shown in Fig. 5 (red bars). Although the dynamic properties of the samples are not very different, the measured coefficients of friction differ drastically. However, the hysteresis part of the friction is approximately similar for all the samples. This is because hysteresis is dependent on the dynamical properties of the rubber samples (and roughness which is similar in all tribosystems), but the total friction is also dependent on the real area of contact (that is also dependent on viscoelasticity and the roughness of the sample) and the frictional shear stress $\tau_{\mathrm{f}}$, as given in Eq. (1). Therefore, the frictional shear stress should be the source of the difference in the measured total friction for samples (1) and (2).

Another major difference between the two studied tribologiocal systems is the running-in phase: The measured friction coefficient between sample (1) in contact with the rough granite ball becomes constant (steady state) after a short run-in distance and hardly changes anymore; however, the measured friction coefficient decreases gradually and becomes stable only after a long run-in distance for sample (2) as shown in Fig. 6.
Fig. 5 Total and hysteresis component of friction (measured by pin-on-disk test rig) and the run-in distance for samples (1) and (2) before the measured friction is stabilized (Color figure online)
Fig. 6 Measured coefficient of friction and its transient shown versus sliding distance
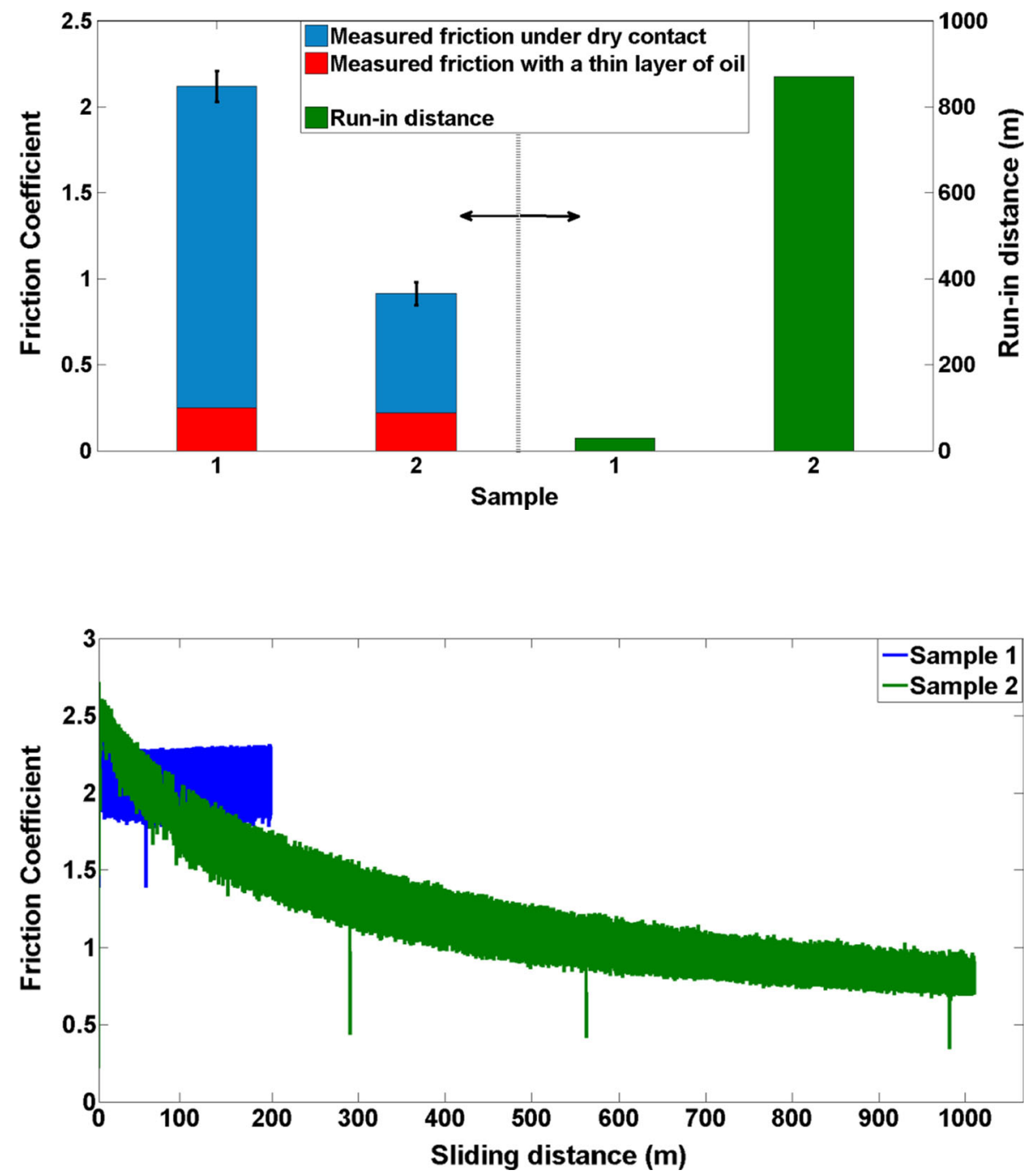


\section{Numerical Results}

The hysteresis coefficient of friction, based on Eq. (2) and using the measured roughness and mechanical properties of the samples, is calculated for both samples and shown as a function of velocity in Fig. 7. The calculated coefficient of friction matches with the measured hysteresis component of friction shown with red bars in Fig. 5. As mentioned before, the hysteresis part of the friction is not very different simply because of their nearly similar mechanical properties.

Moreover, the ratio between the real area of contact and the apparent contact area, for a sliding velocity of $v=5 \mathrm{~mm} / \mathrm{s}$, as a function of the magnification factor $\zeta=q / q_{0}$, is calculated using Eq. (3). The numerical results are presented in Fig. 8.

Considering the fact that sample (1) is elastically softer, it deforms more, and therefore, the calculated real area of contact for sample (1) is higher than for sample (2). However, this difference in the calculated contact area cannot explain the differences shown in the measured friction coefficients. Based on Eq. (5), the shear strength should be taken into account to explain the difference in friction.

\section{AFM Nano-indentations}

As discussed earlier, the contribution to friction from the real area of contact plays an important role in determining the overall friction in the studied tribo-system. Hence, the properties of the top rubber layer in contact with the granite sphere should be studied. There are not many studies where existence of a modified surface layer on top of the rubber has been explored. In [25], scanning electron microscopy (SEM) imaging was used to visualize such a layer. No quantitative data, except about the thickness of the layer, could be derived from SEM imaging; therefore, atomic force microscope (AFM) nano-indentations were used to investigate the mechanical properties of the modified
Fig. 7 Hysteresis coefficient of friction as a function of sliding velocity, calculated for both samples
Fig. 8 Variation of real area of contact over nominal contact area as a function of magnification for sliding velocity of $v=5 \mathrm{~mm} / \mathrm{s}$ for both samples
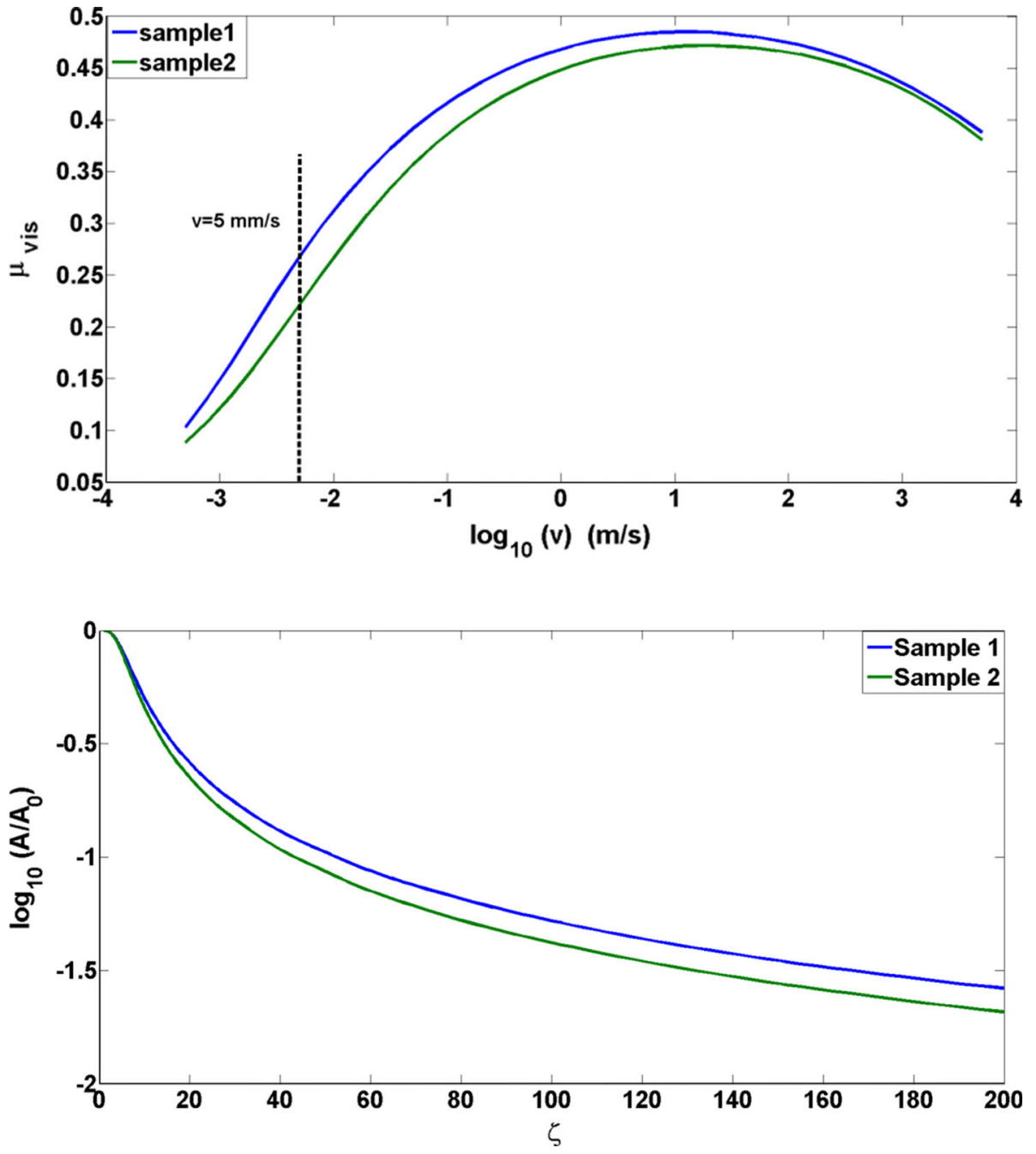
surface layer [20]. The mechanical properties of the wear track of both samples were studied using AFM nano-indentations and compared with the bulk or non-contact sections of the rubber disk. In order to apply low loads and consequently bring about low penetration depths, a cantilever with a low spring constant (a nominal spring constant of $0.10 \mathrm{~N} / \mathrm{m}$ ) was used.

To avoid surface roughness effects in determining the elastic modulus from nano-indentation results, a featureless, smooth region of the rubber was selected. The absolute roughness of the regions selected for nano-indentation was in the order of a few $\mathrm{nm}$ on a $1 \mu \mathrm{m} \times 1 \mu \mathrm{m}$ scale. Further, no evident residual imprint was detected after penetration which suggests no occurrence of plastic deformation due to indentation. Thus, only phenomena with viscoelastic nature are active during indentation. The deflection sensitivity was measured by indenting an elastically hard material, i.e., silicon at various indenter rates. The procedure proposed by Green [29] was used to measure the spring constant of the cantilever. Nano-indentations were performed with loads ranging from about 0.04-187 $\mathrm{nN}$ and indentation rates in the range $21-3.6 \times 10^{8} \mathrm{~nm} / \mathrm{s}$.

The contact model of a blunted pyramidal tip indenting an elastic half-space [30] was used to analyze the forceindentation depth diagrams. Considering the fact that the unloading part of the force-indentation depth diagrams is not advantageous for quantitatively characterizing the studied surfaces [31], the elasticity modulus of the samples was obtained by the loading part of the indentation tests.

The calculated moduli of elasticity for both samples are shown as a function of indenter rate in Fig. 9.

The nano-indentation results do not reveal any changes in the mechanical properties of sample (1), though the wear
Fig. 9 Time dependence of elastic modulus for: a sample (1), b sample (2), both inside and outside the wear track, measured by AFM nanoindentations
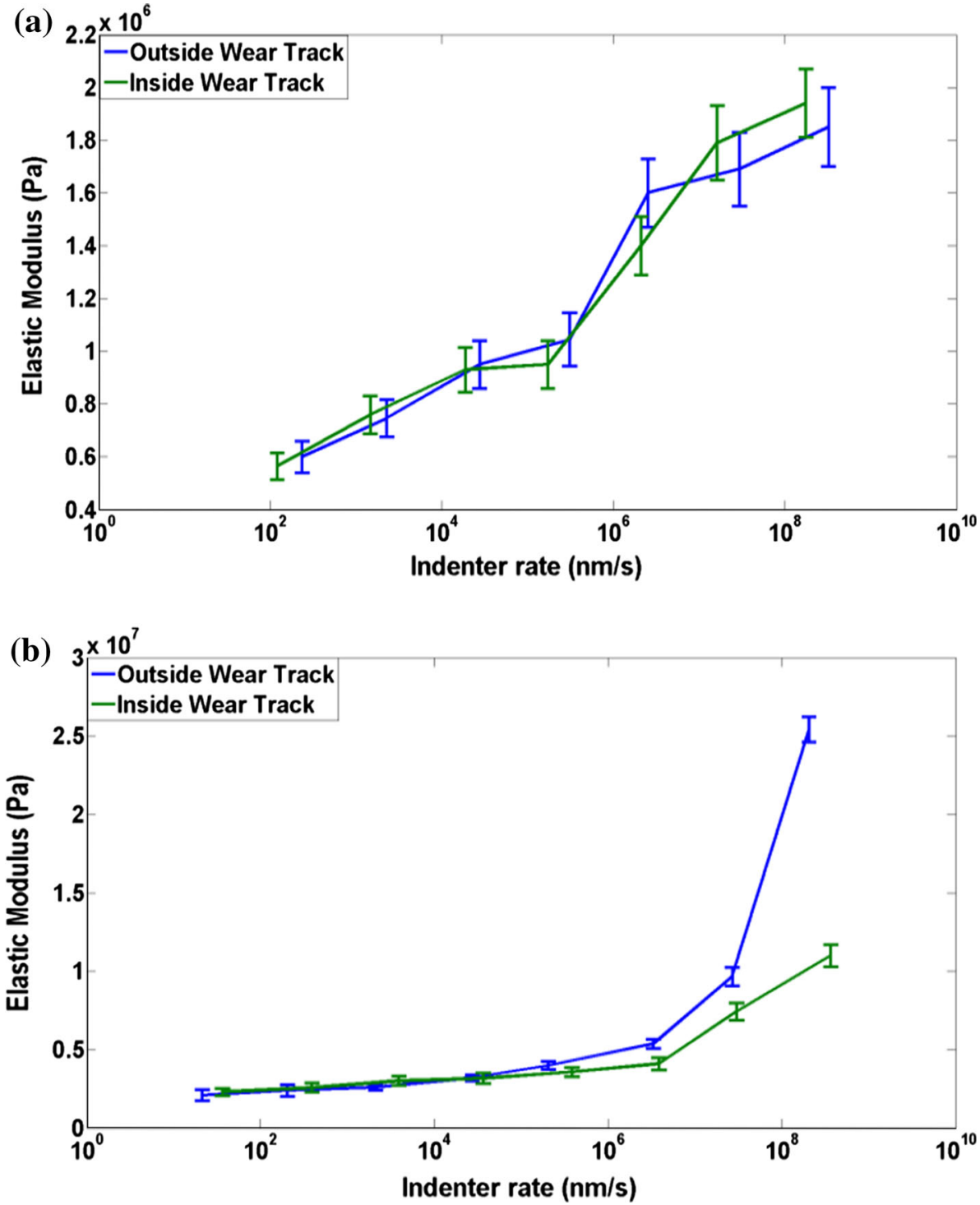
track of sample (2) has different mechanical properties in terms of elastic modulus. This change is more noticeable when indenting the rubber with a higher velocity.

\section{Discussion}

The AFM nano-indentation results presented in Sect. 4 demonstrate the absence of the modified surface layer on sample (1); the elastic moduli inside and outside the wear track are approximately similar. It was shown that formation of such a layer and its properties are dependent on the tribological conditions and consequently the mechanical energy that has been applied to the rubber surface [20]. Considering the fact that the coefficient of friction of sample (1) is higher than for sample (2), it may be concluded that the frictional work generated in contact with sample (1) with the granite is higher in comparison with the sample (2) granite tribo-system. Nonetheless, the frictional work is not only dissipated in tribo-material evolution but also in wear particle generation.

The wear debris of both samples in contact with the granite is powdery and "dusty-like" as shown in Fig. 10. The specific wear rates are $k_{1}=1.83 \times 10^{-1} \mathrm{~mm}^{3} / \mathrm{Nm}$ and $k_{2}=3.87 \times 10^{-3} \mathrm{~mm}^{3} / \mathrm{Nm}$ for samples (1) and (2), respectively.

Sample (1) has a much worse resistance to wear in comparison with sample (2). This is evident, when comparing the specific wear rates which differ by even two decades of magnitude. This can be explained by the concept of the crack mean-free path, using theory of powdery rubber wear [32]; it has been suggested that reducing the crack mean-free path results in reduction of the wear rate. Unreinforced rubber compound, because of lack of (strong) inhomogeneities which can scatter the crack tip and reduce the crack mean-free path, has a very bad wear resistance. On the other hand, in a reinforced rubber when the crack tip reaches a filler particle cluster, it may bend by $\sim 90^{\circ}$ rather than penetrate through the filler particle. More particularly, the smaller wear rate for sample (2) is due to much larger energy (per unit area) required to propagate a crack in the reinforced rubber, as discussed in [33].

The frictional energy can be dissipated by different mechanisms such as frictional heating, elastic (and or plastic) deformations or fracture of one or both bodies in contact, the formation of modified surface layers and tribomaterials, making or breaking adhesive bonds and wear. In the contact between an elastomer and a rigid surface, wear, formation of tribo-modified surface layers and heat generation are the main modes of energy dissipation. In the current study, the sliding velocity is kept low such that heat generation is negligible. Therefore, the main forms of energy dissipation are formation of the modified surface layer and wear. The tribological energy input exerted into the tribo-system is defined as the product of the friction force $F_{\mathrm{f}}$ and worked length $s$.

$E_{\text {in }}=\sum F_{\mathrm{f}} s=F_{\mathrm{N}} \int \mu s \mathrm{~d} s$

The concept of formation and removal of a thin layer with different properties than the bulk of the material, the competition between these two phenomena and the balance between them has been studied for other materials such as ceramics [34] or steel components in the boundary lubrication regime and at presence of lubricant additives [35]. The sliding resistance of an elastomer in contact with a rigid rough surface is dependent on the nature of the interface which is prone to changing because of the frictional energy dissipation during use. Subsequently, wear should be considered in the context of friction of elastomers. The (a)

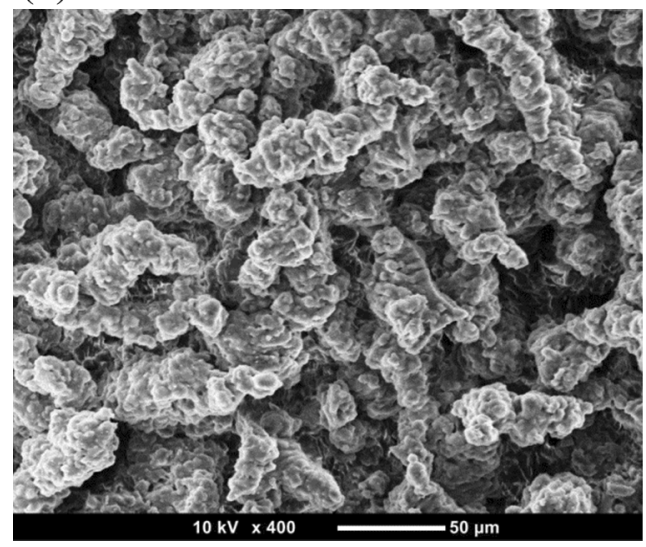

(b)

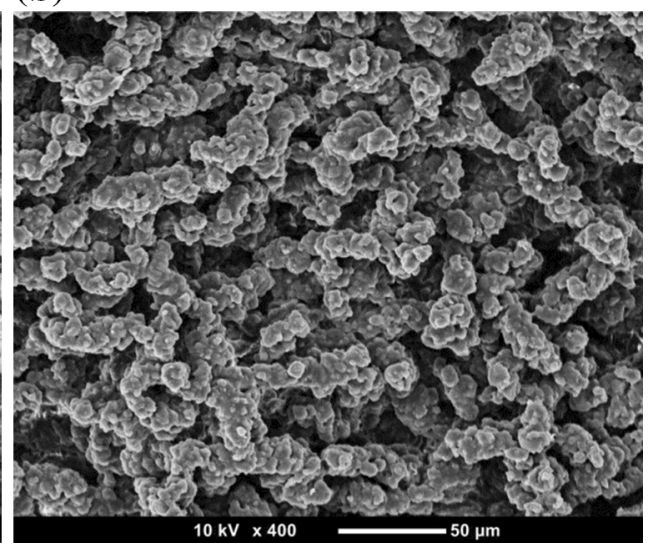

Fig. 10 Scanning electron microscope images of rubber wear particles: a sample (1), b sample (2) 
necessity of embedding wear models into friction models has been shown for other materials [36]. The balance of the modified surface layer writes:

$\frac{\mathrm{d} \delta_{\text {total }}}{\mathrm{d} t}=Q_{\mathrm{f}}-Q_{\mathrm{w}}$

where $\delta_{\text {total }}$ is the thickness of the modified surface layer, $Q_{\mathrm{f}}$ is the formation rate of the modified surface layer and the $Q_{\mathrm{w}}$ is the wear rate. Under steady-state conditions, $\frac{\mathrm{d} \delta_{\text {total }}}{\mathrm{d} t}=0$, and therefore, $Q_{\mathrm{f}}=Q_{\mathrm{w}}$. More experimental studies are required to model the formation of the modified surface layer theoretically, and therefore, the formation rate is not studied in the present study. However, it is known that the formation rate is a function of the tribological conditions [20] (normal pressure, sliding velocity, roughness and temperature). Wear can be addressed by Archard's formulation [37]:

$Q_{\mathrm{w}}=k \sigma_{0} v$ where the wear rate is directly proportional to the applied pressure $\sigma_{0}$ multiplied by the sliding speed $v$. The proportionality constant $k$ is dependent on the normal pressure $\sigma_{0}$. The balance presented in Eq. (7) can also be written as a balance in thickness: $\delta_{\text {total }}=\delta_{\mathrm{f}}-\delta_{\mathrm{w}}, \delta_{\mathrm{w}}=$ $k\left(\sigma_{0}\right) \sigma_{0} s, \delta_{\mathrm{f}}=f\left(T, \sigma_{0}, R, v\right)$. Two different conditions are conceivable: (1) $\delta_{\mathrm{f}}>\delta_{\mathrm{w}}$ and (2) $\delta_{\mathrm{f}} \leq \delta_{\mathrm{w}}$. Existence of a tribo-modified surface layer and the frictional energy of the tribo-system can be correlated as illustrated in Fig. 11. $\delta_{\mathrm{f}}$ and $\delta_{\mathrm{w}}$ are shown schematically in Fig. 11a, and $\delta_{\text {total }}$ which is the difference between the thicknesses of the formed modified surface layer and the worn layer is shown in Fig. 11b. Three different conditions are explained using Fig. 11b; the rubber is exposed to wear as soon as it becomes in contact with a counter-surface. However, in order to generate a thin modified surface layer, sufficient amount of energy should be exerted to the rubber surface. This is shown schematically in Fig. 11 where the thickness of the
Fig. 11 Schematic division of the exerted energy to the rubber surface (in the form of frictional energy) into different situations regarding existence of a modified surface layer (Color figure online)

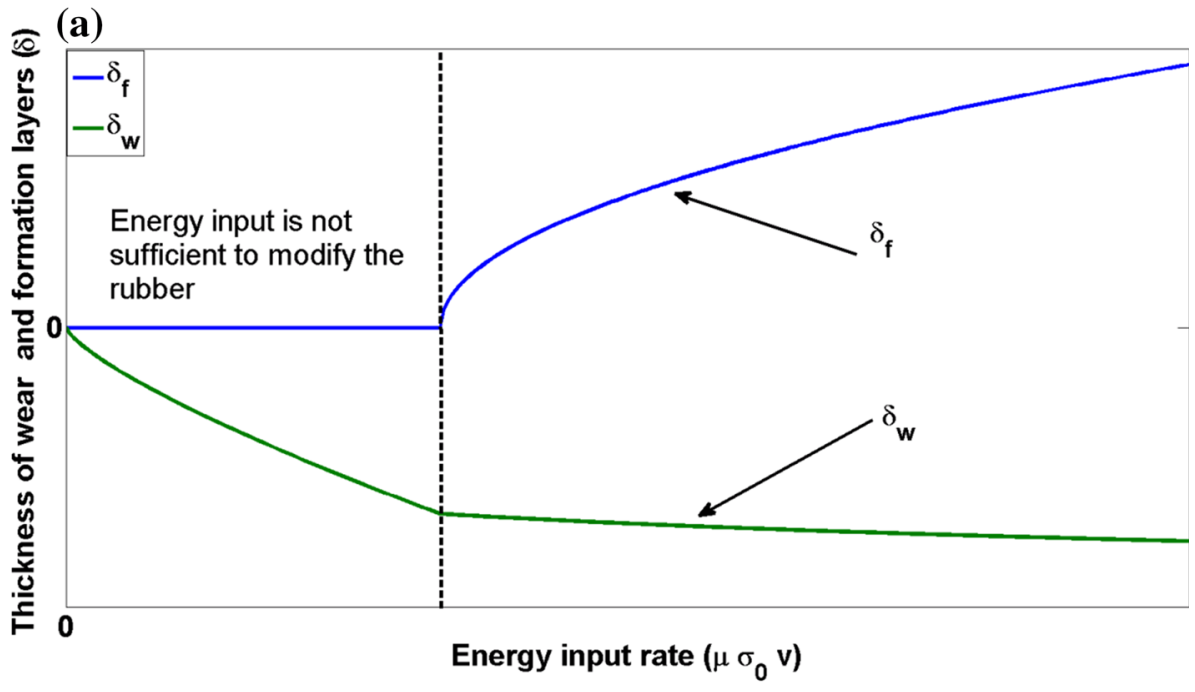

(b)

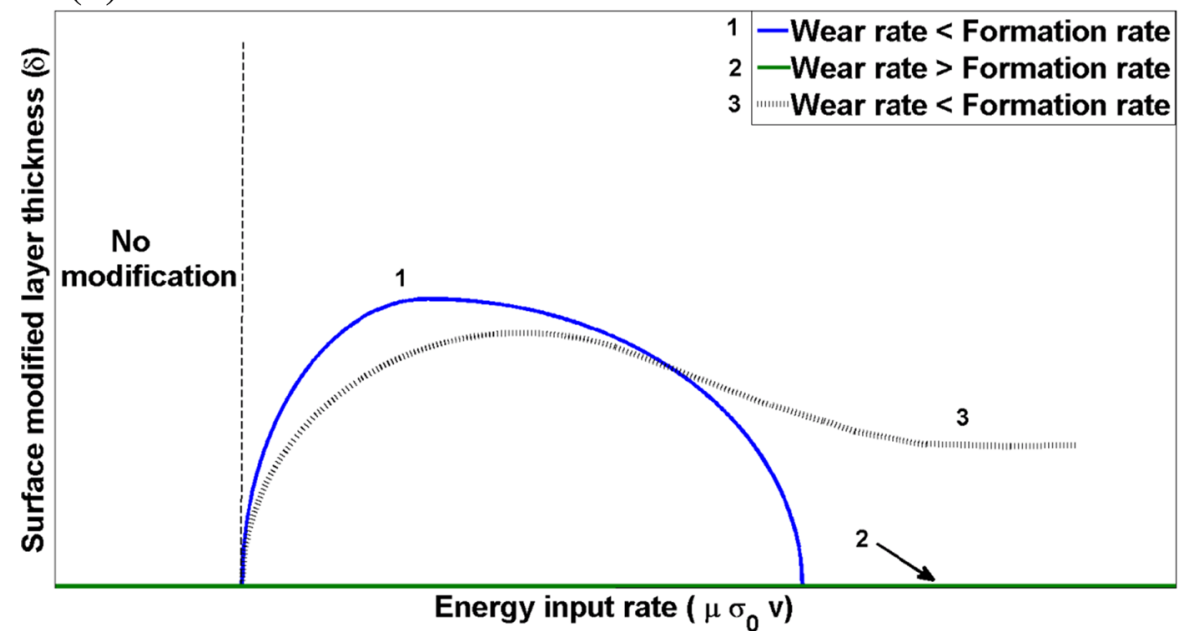


formed modified surface layer is nonzero only when the energy input rate is higher than a minimum value required to modify the surface. This energy level differs for different compounds. The thickness of the formed modified surface layer and the layer thickness worn away both increase by an increase in the energy input. Yet, the existence of the modified surface layer depends on the ratio between $\delta_{\mathrm{f}}$ and $\delta_{\mathrm{w}}$. If the wear rate is higher than the formation rate [condition (2)], the whole modified layer is worn, and therefore, no modified surface layer is present (this condition is demonstrated using green line in Fig. 11b). However, if $\delta_{\mathrm{f}}>\delta_{\mathrm{w}}$, the modified surface layer exists. Under this condition, an increase in energy input rate (which increases both $\delta_{\mathrm{f}}$ and $\delta_{\mathrm{w}}$ ) can bring about two conditions; as shown in Fig. 11b, the first situation is that with an increase in energy input, the thickness of the layer worn away increases more than the thickness of the formed modified surface layer, and therefore, the total modified surface layer thickness will decrease and will be completely removed (blue line). The second situation is when both thicknesses increase proportionally such that the modified surface layer exists for higher energy input rates (black line).

Considering the size of typical stones used in asphalt roads in comparison with the typical thickness of the modified surface layers on rubbers [25], the modified surface layer does not change the hysteresis part of the friction remarkably. This can be shown theoretically [38] and has also been proven experimentally (compare Sects. 2.3 and 3 ). Nonetheless, the modified surface layer can notably change the contribution from real area of contact by alteration of the shear stress and, consequently, the overall friction. Accordingly, the existence and the mechanical properties of the modified surface layer are crucial for correctly modeling friction.

The difference in wear rates can explain the differences seen in the measured friction coefficients. Consider Fig. 6, where the measured friction for sample (1) is stabilized and does not change anymore just after a short sliding distance. Conversely, the measured friction signal stabilizes after a much longer distance for the reinforced sample (2). Because of the high wear rate of sample (1) in contact with the granite counter-surface, the tribo-system does not find the opportunity to form a modified surface layer. Therefore, no significant difference is seen in mechanical properties of the wear track and outside the wear track for sample (1). However, a competition occurs between generation and wear of the modified layer in the contact between sample (2) and the rigid rough counter-surface, in the run-in phase, till the formation and wear rate of such a layer are in balance. As a result of such a balance, the measured friction stabilizes (note the difference in run-in distance, presented by green bars in Fig. 5). Hence, the main condition for the existence of a modified surface layer is the balance between the formation and wear rate of it.

\section{Summary and Conclusions}

The composition and tribological properties of a rubber-rigid surface interface are subject to change during use, especially under dry and boundary lubrication conditions. Although the changes of the interfaces and formation of the tribo-films have been studied for different materials, research on modified rubber surfaces due to interaction between the rubber surface and counter-surface did not receive much attention. The existence of a modified surface layer on rubbers in contact with a rigid rough surface has been shown [20, 25]; however, the dynamics of the modified surface layer formation, surface layer removal and the stability of the modified surface layer is still not satisfactorily studied.

A current perception of the removal and the stability of the modified surface layers on rubbers are introduced alongside experimental work and discussion of the literature. Two rubber samples were prepared, and their dynamic mechanical properties were measured using DMA. The mechanical properties of the samples and thereupon the hysteresis contribution to the friction do not differ much; however, their measured friction in contact with a granite rough surface showed a clear difference. Both theory and experiments validated that the hysteresis part of the friction is not the source of such difference. AFM nanoindentations showed that a modified surface layer with different mechanical properties from the bulk of the material does exist on the wear track of sample (2). However, no such layer could be identified on sample (1). Moreover, the specific wear rate of sample (1) is two decades of magnitude higher than for sample (2). It has been concluded that the existence of a modified surface layer and its definitive role on friction is not only determined by the tribological conditions [20], but that wear also plays a crucial role in this argument. A conceptual model is presented, dividing the energy input to the rubber surface to different zones concerning the existence of a tribo-film. The model suggests that the modified surface layer is formed if only the energy input is sufficient to make such a surface modification. An increase in the input energy results in a more degradation of the rubber; however, more increase in energy input might result in excessive wear of the modified layer so that no modified layer remains. In such a way, the decisive importance of wear on the existence of a modified surface layer is demonstrated.

Open Access This article is distributed under the terms of the Creative Commons Attribution License which permits any use, distribution, and reproduction in any medium, provided the original author(s) and the source are credited. 


\section{References}

1. Grosch, K.A.: The relation between the friction and visco-elastic properties of rubber. Proc. R. Soc. Lond. A 274(1356), 21-39 (1963)

2. Persson, B.N.J.: Theory of rubber friction and contact mechanics. J. Chem. Phys. 115(8), 3840-3861 (2001)

3. Bódai, G., Goda, T.J.: Friction force measurement at windscreen wiper/glass contact. Tribol. Lett. 45(3), 515-523 (2012)

4. Mofidi, M., et al.: Rubber friction on (apparently) smooth lubricated surfaces. J. Phys. Condens. Matter. 20(8) (2008). doi:10. 1088/0953-8984/20/8/085223

5. Moore, D.F.: The Friction and Lubrication of Elastomers, vol. 9, 288 pp. Pergamon Press, Oxford, New York (1972)

6. Wriggers, P., Reinelt, J.: Multi-scale approach for frictional contact of elastomers on rough rigid surfaces. Comput. Methods Appl. Mech. Eng. 198(21-26), 1996-2008 (2009)

7. Lorenz, B., et al.: Rubber friction: comparison of theory with experiment. Eur. Phys. J. E 34(12) (2011)

8. Lorenz, B., et al.: Rubber friction for tire tread compound on road surfaces. J. Phys. Condens. Matter 25(9) (2013). doi:10.1088/ 0953-8984/25/9/095007

9. Pan, X.D.: Wet sliding friction of elastomer compounds on a rough surface under varied lubrication conditions. Wear 262(5-6), 707-717 (2007)

10. Greenwood, J.A., Williamson, J.B.P.: Contact of nominally flat surfaces. Proc. R. Soc. Lond. A 295(1442), 300-319 (1966)

11. Putignano, C., et al:: A new efficient numerical method for contact mechanics of rough surfaces. Int. J. Solids Struct. 49(2), 338-343 (2012)

12. Haines, D.J., Ollerton, E.: Contact stress distributions on elliptical contact surfaces subjected to radial and tangential forces. Proc. Inst. Mech. Eng. 177(1), 95-114 (1963)

13. Paggi, M., Ciavarella, M.: The coefficient of proportionality $\kappa$ between real contact area and load, with new asperity models. Wear 268(7-8), 1020-1029 (2010)

14. Afferrante, L., Carbone, G., Demelio, G.: Interacting and coalescing Hertzian asperities: a new multiasperity contact model. Wear 278-279, 28-33 (2012)

15. Yastrebov, V.A., Anciaux, G., Molinari, J.-F.: From infinitesimal to full contact between rough surfaces: evolution of the contact area. Int. J. Solids Struct 52, 83-102 (2015)

16. Manners, W., Greenwood, J.A.: Some observations on Persson's diffusion theory of elastic contact. Wear 261(5-6), 600-610 (2006)

17. Dapp, W.B., Prodanov, N., Müser, M.H.: Systematic analysis of Persson's contact mechanics theory of randomly rough elasticsurfaces. J. Phys. Condens. Matter 26(35) (2014). doi:10.1088/ 0953-8984/26/35/355002

18. Jacobson, S., Hogmark, S.: Surface modifications in tribological contacts. Wear 266(3-4), 370-378 (2009)

19. Mokhtari, M., Schipper, D., Tolpekina, T.: On the friction of carbon black- and silica-reinforced BR and S-SBR elastomers. Tribol. Lett. 54(3), 297-308 (2014)
20. Mokhtari, M., Schipper, D.J.: Existence of a tribo-modified surface layer of BR/S-SBR elastomers reinforced with silica or carbon black. Tribol. Int. (2014). doi:10.1016/j.triboint.2014.09. 021

21. Martínez, L., et al.: Influence of friction on the surface characteristics of EPDM elastomers with different carbon black contents. Tribol. Int. 44(9), 996-1003 (2011)

22. Degrange, J.M., et al.: Influence of viscoelasticity on the tribological behaviour of carbon black filled nitrile rubber (NBR) for lip seal application. Wear 259(1-6), 684-692 (2005)

23. Karger-Kocsis, J., et al.: Dry friction and sliding wear of EPDM rubbers against steel as a function of carbon black content. Wear 264(3-4), 359-367 (2008)

24. Nakazono, T., Matsumoto, A.: Mechanical aging behavior of styrene-butadiene rubbers evaluated by abrasion test. J. Appl. Polym. Sci. 120(1), 379-389 (2011)

25. Rodríguez, N.V., Masen, M.A., Schipper, D.-J.: Tribologically modified surfaces on elastomeric materials. Proc. Inst. Mech. Eng. Part J. J. Eng. Tribol. 227, 398-405 (2013)

26. Wang, M.-J., Kutsovsky, Y.: Effect of fillers on wet skid resistance of tires. Part I: water lubrication vs. filler-elastomer interactions. Rubber Chem. Technol. 81(4), 552-575 (2008)

27. Wang, M.-J., Kutsovsky, Y.: Effect of fillers on wet skid resistance of tires. Part II: experimental observations on effect of filler-elastomer interactions on water lubrication. Rubber Chem. Technol. 81(4), 576-599 (2008)

28. Persson, B.N.J.: On the fractal dimension of rough surfaces. Tribol. Lett. 54(1), 99-106 (2014)

29. Green, C.P., et al.: Normal and torsional spring constants of atomic force microscope cantilevers. Rev. Sci. Instrum. 75(6), 1988-1996 (2004)

30. Rico, F., et al.: Probing mechanical properties of living cells by atomic force microscopy with blunted pyramidal cantilever tips. Phys. Rev. E 72(2), 021914 (2005)

31. Tranchida, D., Piccarolo, S.: On the use of the nanoindentation unloading curve to measure the young's modulus of polymers on a nanometer scale. Macromol. Rapid Commun. 26(22), 1800-1804 (2005)

32. Persson, B.N.J.: Theory of powdery rubber wear. J. Phys.: Condens. Matter 21(48), 485001 (2009)

33. Persson, B.N.J.: Model study of brittle fracture of polymers. Phys. Rev. Lett. 81(16), 3439-3442 (1998)

34. Valefi, M., et al.: Modelling of a thin soft layer on a self-lubricating ceramic composite. Wear 303(1-2), 178-184 (2013)

35. Morina, A., Neville, A.: Understanding the composition and low friction tribofilm formation/removal in boundary lubrication. Tribol. Int. 40(10-12), 1696-1704 (2007)

36. Blau, P.: Embedding wear models into friction models. Tribol. Lett. 34(1), 75-79 (2009)

37. Archard, J.F.: Contact and rubbing of flat surfaces. J. Appl. Phys. 24(8), 981-988 (1953)

38. Persson, B.N.J.: Contact mechanics for layered materials with randomly rough surfaces. J. Phys. Condens. Matter 24(9) (2012). doi:10.1088/0953-8984/24/9/095008 